\title{
COMPUTATIONAL METHODS FOR BIOMEDICAL IMAGE PROCESSING AND ANALYSIS
}

\section{Automatic tracking of labeled red blood cells in microchannels}

\author{
Diana Pinho $^{1,2, *, \dagger}$, Rui Lima ${ }^{1,3}$, Ana I. Pereira ${ }^{1,4}$ and Fernando Gayubo ${ }^{5}$ \\ ${ }^{1}$ Institute Polytechnic of Bragança, Portugal \\ ${ }^{2}$ FEUP, Porto University, Portugal \\ ${ }^{3}$ CEFT, FEUP, Porto University, Portugal \\ ${ }^{4}$ Algoritmi, University of Minho, Portugal \\ ${ }^{5}$ Fundación CARTIF, Valladolid, Spain
}

\begin{abstract}
SUMMARY
The current study proposes an automatic method for the segmentation and tracking of red blood cells flowing through a $100-\mu \mathrm{m}$ glass capillary. The original images were obtained by means of a confocal system and then processed in MATLAB using the Image Processing Toolbox. The measurements obtained with the proposed automatic method were compared with the results determined by a manual tracking method. The comparison was performed by using both linear regressions and Bland-Altman analysis. The results have shown a good agreement between the two methods. Therefore, the proposed automatic method is a powerful way to provide rapid and accurate measurements for in vitro blood experiments in microchannels. Copyright (C) 2012 John Wiley \& Sons, Ltd.
\end{abstract}

Received 15 February 2012; Revised 21 September 2012; Accepted 11 October 2012

KEY WORDS: image analysis; automatic method; red blood cells; microcirculation; in vitro blood flow; microchannels

\section{INTRODUCTION}

Blood flow in microcirculation is crucial for the normal function of tissues and organs. Detailed study of blood cells flowing in microvessels and microchannels is essential to provide a better understanding on the blood rheological properties and disorders in microcirculation [1-9]. One of the most reliable ways to measure velocity fields in microcirculation is using Eulerian methods, such as the conventional micro-Particle Image Velocimetry (PIV) [10-17] or the confocal micro-PIV $[1,5,8,9]$. By using these Eulerian methods, the most common methodology to calculate the velocity fields is a statistical technique known as cross-correlation. For high concentration of particles, that is, the interrogation window contains at least three particles, the micro-PIV technique is the best suitable methodology to study blood flow phenomena in microcirculation. However, most of in vivo measurements contain physiological fluids with high concentrations of blood cells and as a result the amount of tracer particles captured within the fluid is often very low [8]. If the number of particles or cells within the interrogation area is small (less than three), it is recommended to measure the displacements of each individual object by tracking them in a Lagrangian way [5]. This kind of PIV mode (low-image-density PIV) is often referred to as particle tracking velocimetry (PTV) $[5,14,15]$. The main advantage of this method is the ability to obtain detailed quantitative information on the motion of particles and cells flowing within a working fluid with high hematocrits (Hcts) close to in vivo condition. Although, this method is becoming indispensable, in several biomedical fields

*Correspondence to: Diana Pinho, Instituto Politécnico de Bragança / ESTiG, Campus de Santa Apolónia Apartado 1134, 5301-857 Bragança, Portugal.

†E-mail: diana@ipb.pt 
such as cell biology [16,17] and microcirculation [1,5,6,8,9,14-22], most of these studies have been performed with manual tracking methods. For blood flow in microvessels, where there is a large number of interacting cells, manual tracking methods have been used to accurately track individual deformable cells flowing through glass capillaries $[1,16,19,20]$, straight polydimethylsiloxane microchannels [22], stenosis [23], hyperbolic contractions [24, 25], and bifurcations [26, 27]. However, the manual data collection is extremely time-consuming to have a statistically representative number of samples and may introduce operators' errors that eventually limit the application of these methods many times at different conditions. Hence, it is crucial to develop a versatile and automatic tracking method able to compute automatically multiple cell trajectories and remove errors by the user's evaluation. Several researchers have been developing different kinds of automatic algorithms for Image J [28], MATLAB [29, 30], LabVIEW [31], and IDL [32]. A promising PTV plug-in for Image $\mathbf{J}$ is the 'Particletracker 2D and 3D' [15]. However, this plug-in is still under development because the trajectory results tend to overlap at high Hcts.

Despite the great effort of many researchers, the use of automatic methods to accurately investigate the individual blood cell's motion in microchannels has never been achieved before. This was accomplished in this study by using an automated image processing technique able to track accurately individual red blood cells (RBCs) flowing through a $100-\mu \mathrm{m}$ microchannel. This method can be further adapted for other applications to quantitatively evaluate deformable RBCs and white blood cells flowing in both in vitro and in vivo environments with Hcts lower than $12 \%$.

Image analysis and processing is a huge area that provides a large number of viable applications; one example is the improvement of pictorial information for human interpretation or in the computer-automated analysis. Segmentation is the most used procedure in image processing and consists in dividing a digital image into multiple regions, based on a set of pixels or objects, to simplify and/or change the representation of an image, facilitating their analysis [33,34]. A variety of techniques can be applied. There are simple methods (such as thresholding and masking) or complex methods (such as edge/boundary detection and region growing). The literature contains hundreds of segmentation techniques [35,36], but there is no single method that can be considered good enough for all kinds of images. Segmentation methods change according to the imaging modality, application domain, method being automatic or semiautomatic, and other specific factors. Depending on the image quality and the general image artifacts, such as noise, some segmentation methods may require image preprocessing prior to the segmentation algorithm [37]. On the other hand, some other methods apply post-processing to overcome the problems arising from over segmentation. Overall, segmentation methods can be broadly categorized into point-based, edge-based, and region-based methods $[33,34,37,38]$.

The segmentation of each pixel in the same region is similar with reference to some characteristic or property computation, such as color, intensity, texture or continuity [33]. The process oriented to the analysis of pixels classifies each image point according to the characteristics of color or intensity. Generally, the analyses per pixel are easily implemented in the segmentation. In the thresholding process each pixel in a grayscale image is recognized as either an object or background. A more advanced method creates histograms, oriented to the intensity of grayscale or color, showing the frequency of occurrence of certain intensities in an image. The regions and objects are recognized from these data $[33,34,37,38]$.

The main purpose of this work is to develop an automatic approach to track the RBCs flowing in a microchannel. To accomplish it we tested filtering, segmentation and feature extraction functions available in MATLAB and implemented an application able to obtain the center of the cells ( $x$ and $y$ data) automatically. Finally, we executed a statistical comparison between the manual method (MtrackJ) and the proposed automatic method (RBC-DataTracking 1.0).

\section{MATERIALS AND METHODS}

\subsection{Physiological fluid, RBC labeling and microchannel}

The physiological fluid used in this study was a solution of Dextran 40 (Dx40) from Otsuka Medicine, Tokyo, Japan. First the RBCs were separated by centrifugation, and the plasma and 
buffy coat were removed by aspiration. Washing and centrifugation with physiological saline were repeated three times. The washed RBCs were suspended in Dx40 to make a sample with a Hct of $12 \%$ because Dx40 normally offers a good physiological environment for the RBCs. The Hct corresponds to the feed reservoir Hct and it was measured by using a hematocrit centrifuge (Kubota 3220 , Japan), always immediately before each experiment. It is worth mentioning that the in vitro conditions used in the present study are different from actual in vivo conditions. The low Hct of $12 \%$ was selected to obtain images with the best possible quality and consequently to reduce errors during the cell tracking. The blood used in this study was collected from a healthy adult, and heparin was added to prevent coagulation. The sample was stored hermetically at $4^{\circ} \mathrm{C}$ until the experiment was performed at room temperature $\left(25 \pm 2^{\circ} \mathrm{C}\right)$.

The RBCs were fluorescently labeled with a lipophilic carbocyanime derivative dye, chloromethylbenzamido (CM-Dil, C-7000, Molecular Probes) using a procedure previously described elsewhere $[1,8]$. Briefly the RBCs were separated from the bulk blood by centrifugation and aspiration of the plasma and buffy coat and then washed twice with physiological saline. The washed RBCs were then incubated in the dye solution for $30 \mathrm{~min}$ at $37^{\circ} \mathrm{C}$ and then for an additional time at $4^{\circ} \mathrm{C}$ for about $2 \mathrm{~h}$. The fluorescently labeled RBCs were washed to remove any excess dye and suspended with Dx40 to make up the required RBCs concentration by volume. This dye was well retained by the RBCs and had a strong light intensity, which have allowed a good visualization and tracking labeled RBCs flowing in concentrated suspensions.

The microchannel used in this study was a $100-\mu \mathrm{m}$ circular borosilicate glass capillary fabricated by Vitrocom (Mountain Lakes, NJ, USA). The capillary was mounted on a slide glass with a thickness of $170 \pm 20 \mu \mathrm{m}$ and was immersed in glycerine to minimize the refraction from the walls (see Figure 1).

\subsection{Experimental set-up}

The confocal system used in this study consists of an inverted microscope (IX71; Olympus) combined with a confocal scanning unit (CSU22; Yokogawa), a diode-pumped solid-state (DPSS) laser (Laser Quantum) with an excitation wavelength of $532 \mathrm{~nm}$ and a high-speed camera (Phantom v7.1; Vision Research). The laser beam was illuminated from below the microscope stage through a dry $40 \times$ objective lens with a numerical aperture (NA) equal to 0.9 . The glass capillary was placed on the stage of the inverted microscope, and by using a syringe pump (KD Scientific) a pressuredriven flow was kept constant $(R e \sim 0.008)$. The $R e$ and associated experimental parameters are summarized in Table I.

The light emitted from the fluorescent flowing RBCs passes through a color filter into the scanning unit CSU22, where by means of a dichromatic mirror is reflected onto a high speed camera to

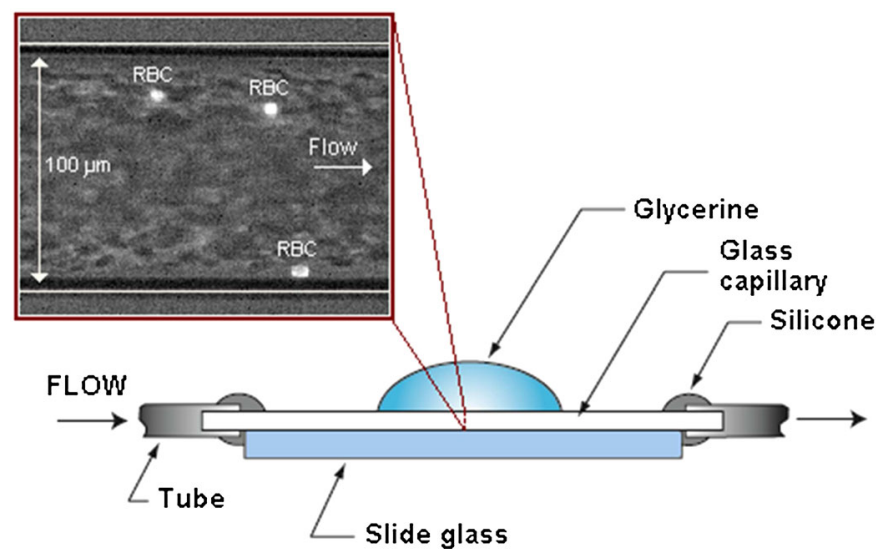

Figure 1. Schematic diagram of the biomedical microdevice used in this study and an image obtained in the center of the microchannel containing both labeled RBCs (bright spots) and no labeled RBCs (gray spots). 
Table I. Experimental parameters used to calculate the Re.

\begin{tabular}{lccccc}
\hline $\begin{array}{c}\text { Density } \\
\left(\mathbf{k g} / \mathbf{m}^{3}\right)\end{array}$ & $\begin{array}{c}\text { Mean velocity } \\
(\mathbf{m} / \mathbf{s})\end{array}$ & $\begin{array}{c}\text { Diameter } \\
(\mathbf{m})\end{array}$ & $\begin{array}{c}\text { Length } \\
(\mathbf{m m})\end{array}$ & $\begin{array}{c}\text { Viscosity of Dx40 } \\
\left(\mathbf{N s} / \mathbf{m}^{2}\right)\end{array}$ & $\mathbf{R e}$ \\
\hline 1046 & $2.6 \times 10^{-4}$ & $10^{-4}$ & 50 & $4.5 \times 10^{-3}$ & 0.008 \\
\hline
\end{tabular}

record the confocal images. Additionally, by using a thermoplate controller (Tokai Hit) the temperature surrounding the capillary was $37^{\circ} \mathrm{C} \pm 1$. A more detailed description of the confocal system used in this study can be found elsewhere $[1,8,18,20,21]$.

\subsection{Image analysis and processing}

All confocal images were captured around the middle of the capillary with a resolution of $640 \times 480$ pixels, at a rate of 100 frames/s with an exposure time of $4995 \mu \mathrm{s}$ and then digitized and transferred to a computer for evaluation using a Phantom camera control software (PH607). Two image analysis methods were used in this study: manual method and automatic method.

2.3.1. Manual method. For the case of the confocal micro-PTV measurements, a series of confocal images were evaluated in Image J [28] using the manual tracking MtrackJ plug-in [39]. The motion of the labeled RBCs were manually tracked through successive images using the bright centroid criteria available at the MtrackJ. Using this method, it was possible to track labeled RBCs even when two cells were in near proximity. After obtaining a series of $x$ and $y$ positions (center position of the cells), data were exported for the determination of several physical quantities such as velocity, radial displacement, mean square displacement (MSD) and dispersion coefficient.

2.3.2. Automatic method. The analysis of microscopic images requires essentially two parts: the image processing where we can apply a filter of preprocessing and segmentation for isolating objects of interest (such as RBCs), to simplify further analysis focusing on these objects with more detailed features such as brightness or size.

To start the image analysis it is necessary to load the videos using MATLAB [29] platform; however, the video file with the extension '.cin' captured by the high speed camera is not supported by MATLAB. As a result, we used the Phantom software to convert video footage to the sequence of images in JPEG format. Using this procedure the images are ready to be treated in MATLAB. The first preprocess is the extraction of the region of interest (region between the walls of the microchannel) by cropping out-of-interest regions.

The next step is the segmentation of the object (RBC). However, first a preprocessing filter, median filter, was applied with one mask of $5 \times 5$ pixel, to smooth the original image and to enhance the flowing object. This filter has performed particularly well in situations where the image is contaminated by impulsive noise (salt-and-pepper) $[16,33,37]$. Noise or artifacts often appear during the flow. Sometimes we can observe cells on other planes of focus or other kinds of cells such as white blood cells. Other sources of noise can be the light absorbed and scattered by the neighboring cells because of the high concentration of cells. The tridimensional motion of the cells is also an important source of noise.

In Figure 2(b) we can see the result of this step; the background is more smoothed and the objects are more defined and visible.

Then, the images are subject to a segmentation step. With this segmentation, only the objects of interest, in this case RBCs, are separated from the background. This is possible by using a threshold method, where a definition of one or more values of separation is enough to divide the image into one or more regions. Mathematically, the operation thresholding can be described as an image processing technique in which an input image $f(x, y)$ of $N$ gray levels produces an image $g(x, y)$, called thresholded image, in which the number of gray levels is less than $N$. An iterative threshold was applied for the sequence of all the images, that is, the threshold level is different from image 

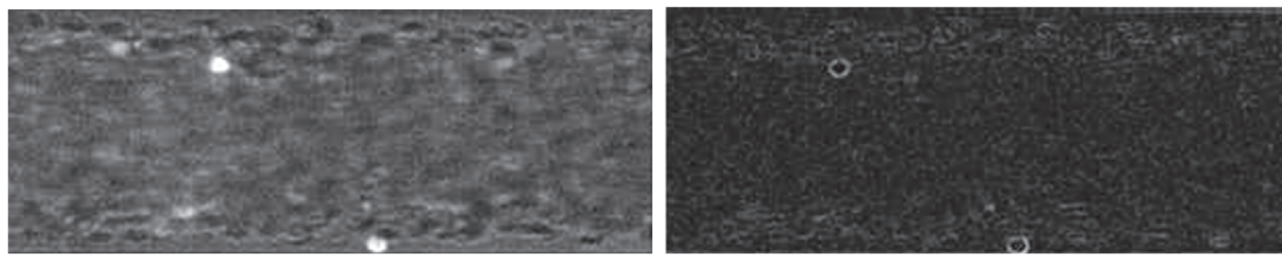

a)
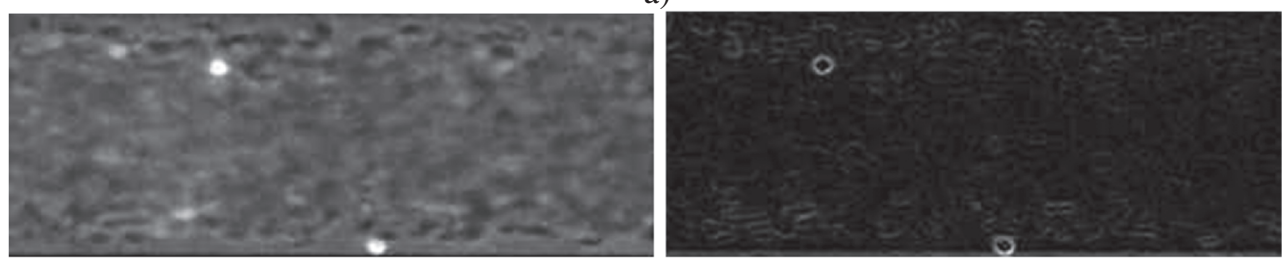

b)

Figure 2. Preprocessing of images: (a) original image (left side) and respective gradient (right side);

(b) original image filtered by using the median filter (left side) and respective gradient (right side).

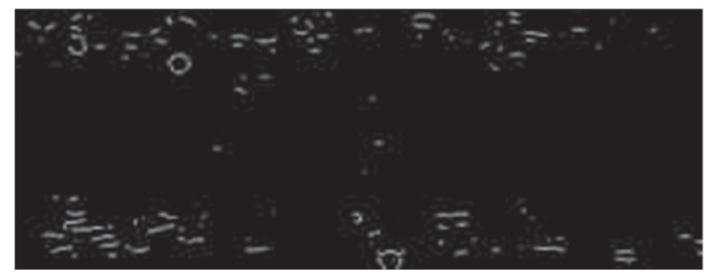

a)

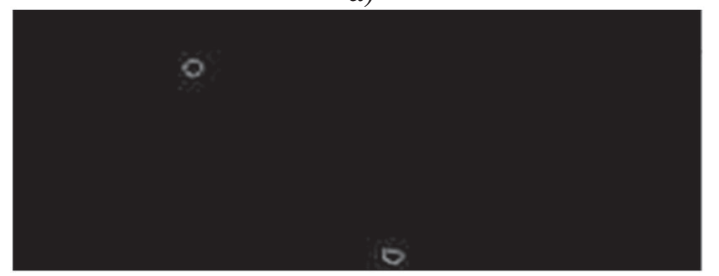

b)

Figure 3. (a) Automatic segmentation before applying Median filter. (b) RBCs tracking and data extraction used in the application proposed.

to image depending on its quality. After thresholding, Sobel filter was applied to improve the visualization of the objects, showing only the edge of the RBCs (see Figure 3). The Sobel function presented in the toolbox Image Processing, from MATLAB, has the ability to find edges using the Sobel approximation to the derivative, that is, it returns edges at those points where the gradient of the image is maximum $[40,41]$.

After the segmentation process, the RBCs were automatically tracked and sets of data ( $x$ and $y$ positions) were obtained. In this step it is also possible to measure a set of properties (area, centroid, etc.) for each connected component (RBC) in the binary image. In our study, sets of centroid positions were mainly used to measure the RBCs trajectories.

2.3.3. Statistical analysis. A statistical software package, MedCal [42, 43], was used for the statistical analyses including linear regression analysis. The Bland-Altman analysis was also performed to compare measurements obtained between the two different methods (manual and automatic method). The error bars on the figures denote sample standard deviation and in all analyses a $95 \%$ of confidence interval was considered. 


\section{RESULTS AND DISCUSSION}

A series of time-consecutive images were obtained by using a confocal system. The measurements were performed at the center plane of a glass microchannel with a diameter of $100 \mu \mathrm{m}$, temperature of approximately $37^{\circ} \mathrm{C}$ and Hct of about $12 \%$. By measuring the radial displacements of 22 labeled RBCs flowing through the microchannel as a function of time, it was possible to calculate the ensemble average of the MSD. The MSD is a statistical quantity that contains information about the RBC tendency to undergo transversal displacements because of multiple interactions with neighboring RBCs. It measures the average distance that an $\mathrm{RBC}$ travels transversally over a certain interval of time and it depends on how often the RBCs suffer collisions. As a result the MSD measures the fluctuations in the trajectories for each individual RBC. Fluctuations in radial position for each RBC can be described in terms of the MSD by using the following equation:

$$
M S D=\left\langle\left(R_{i, y}(t)-R_{i, y}(0)\right)^{2}\right\rangle
$$

where $R_{i, y}$ and $t$ are the radial displacements and time interval, respectively. For a sufficiently long time, the MSDs tend to increase linearly with time and consequently the dispersion coefficient reaches a constant value, which is characteristic of a diffusive regime. The slope of the linear fit to this part of the curve can be used to calculate the corresponding dispersion coefficient $\left(D_{y y}\right)$ $[1,20,22]$

$$
D_{y y}=\frac{1}{N} \sum_{i=1}^{N} \frac{M S D}{2 t}
$$

where $N$ are the number of measured RBCs.

To analyze the ability of the proposed automatic method to track accurately individual labeled RBCs, 22 RBCs trajectories measured by using both manual and automatic tracking methods were used for comparison.

Figure 4 shows the trajectories of individual labeled RBCs, flowing in the center plane of the microchannel, determined by a manual tracking method (MtrackJ, plug-in from Image J) [39] and a proposed automatic tracking method. In these particular cases the mean difference of the first cell (cell 1) is $0.47 \mu \mathrm{m}$ with a standard deviation of 0.12 at $95 \%$ confidence interval whereas for the second cell (cell 2) the mean difference is $0.39 \mu \mathrm{m}$ with a standard deviation of 0.10 at $95 \%$ confidence interval.

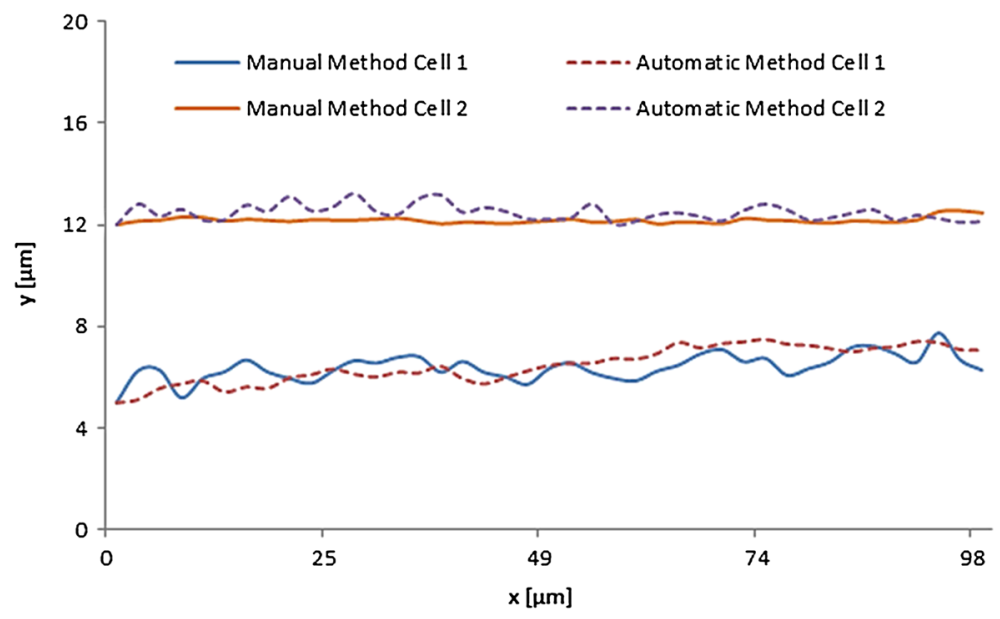

Figure 4. Trajectories of individual labeled RBCs determined by a manual tracking method (MtrackJ, plug-in from Image J) and a proposed automatic tracking method. The measurements were performed in center plane of the microchannel for a feed Hct of $12 \%$. 

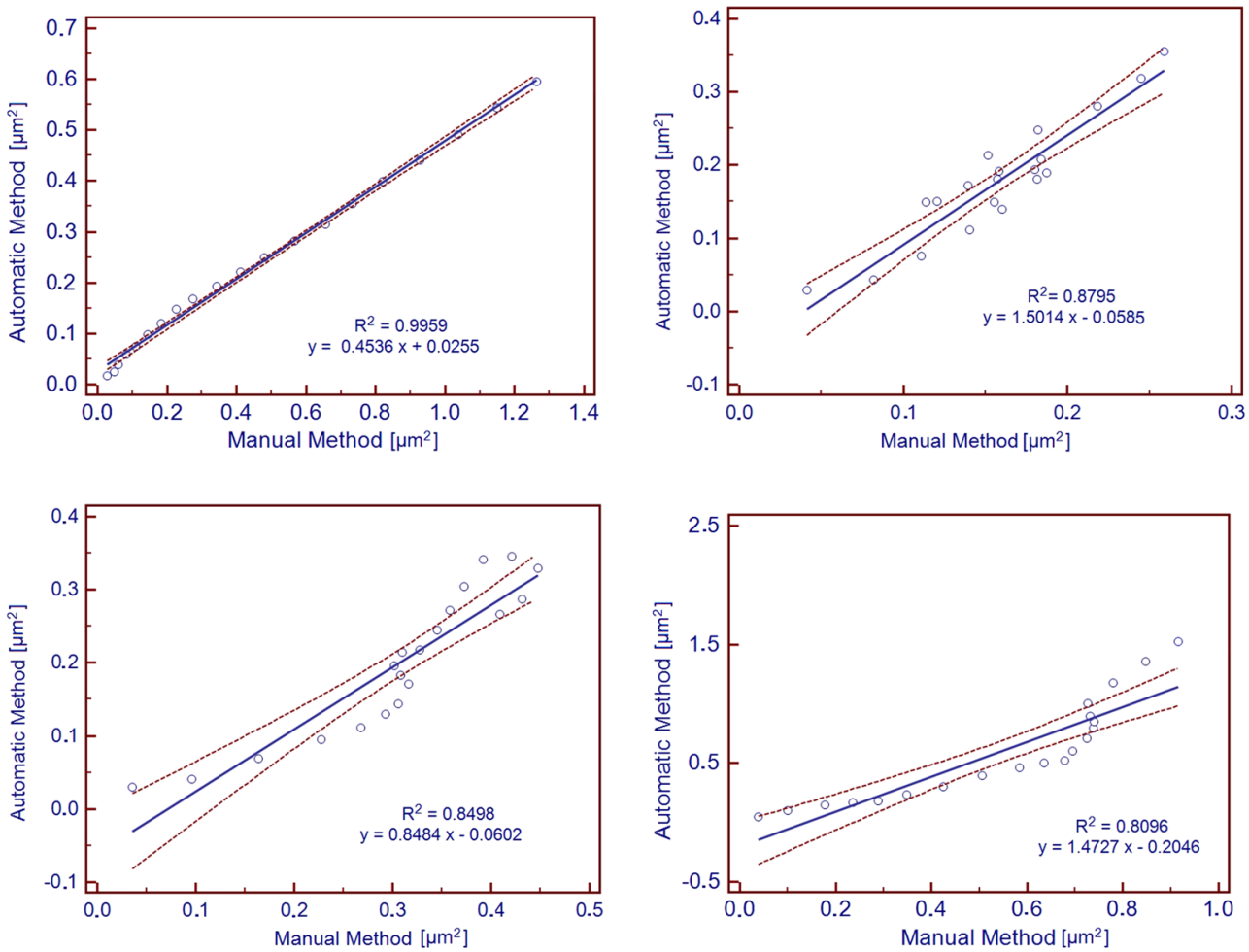

Figure 5. Statistical comparison of the values determined by the manual and automatic methods. Linear regression analysis for four representative measurements, the dashed lines indicate $95 \%$ confidence limits of the measurements.

To examine the accuracy of the proposed automatic method, a linear regression curve and a Bland-Altman analysis were performed by taking the manual measurement as the reference. The analysis was performed using a commercial statistical software package, MedCalc [42, 43]. Figure 5 shows the linear regression curve of the MSD of four representative RBCs for comparison between the manual and automatic measurements. In this study, the correlation coefficient for all the measured cells was between 0.99 and 0.80 indicating a close trend between both methods. We also verified that the slope of the regression line is between 0.45 and 1.50.

Because the linear regression analysis may hide some systematic differences between the manual and automatic methods, for a more detailed statistical comparison the Bland-Altman analysis was also performed. The Bland-Altman analysis provides a more extensive analysis of the agreement between two methods than the linear regression. Furthermore, this is a graphical method to compare two measurement techniques. In this analysis the differences between the manual and automatic methods are plotted against the average of these two methods. If the difference obtained from the two techniques is not large enough and falls within the acceptable limit with a $95 \%$ confidence, then the automatic method is considered to be similar to the manual method. Hence, the BlandAltman method was used to obtain statistical analysis of the agreement between the manual and automatic methods for the RBCs with the best and worst correlation coefficient, 0.9959 and 0.8096 , respectively. Figure 6 shows the linear regression and Bland-Altman results.

The Bland-Altman plot presents the mean difference and agreement limits, which are defined as the mean difference plus and minus 1.96 times the standard deviation of the differences. Because the difference obtained from both methods is not large enough and fell within the acceptable limit with a $95 \%$ confidence, it is possible to conclude that no significant difference between the two methods was found. Therefore, these results indicate a good agreement between the measurements obtained by the manual and automatic methods. 

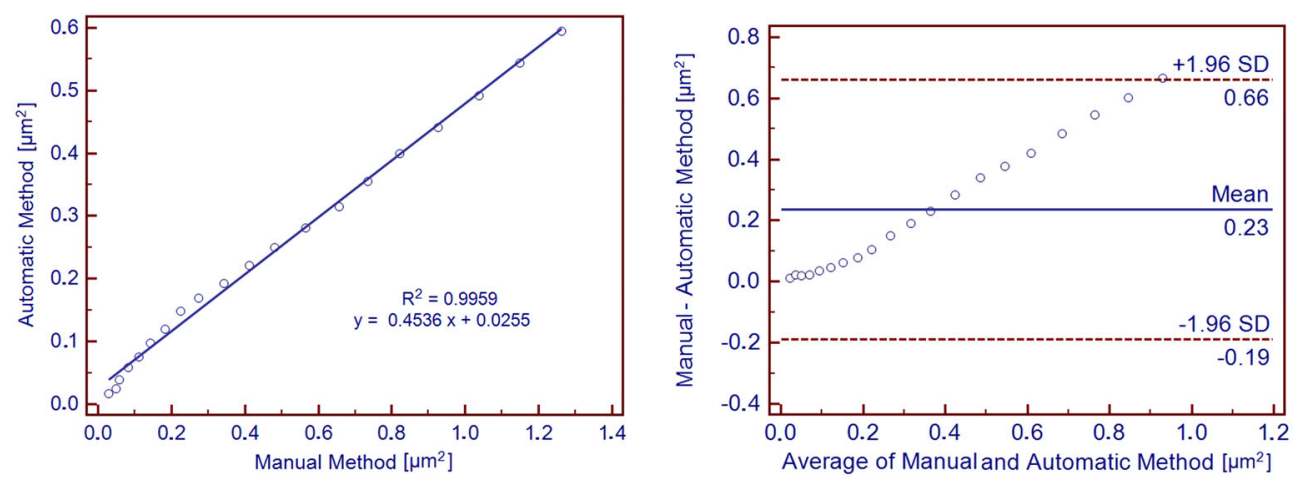

a)
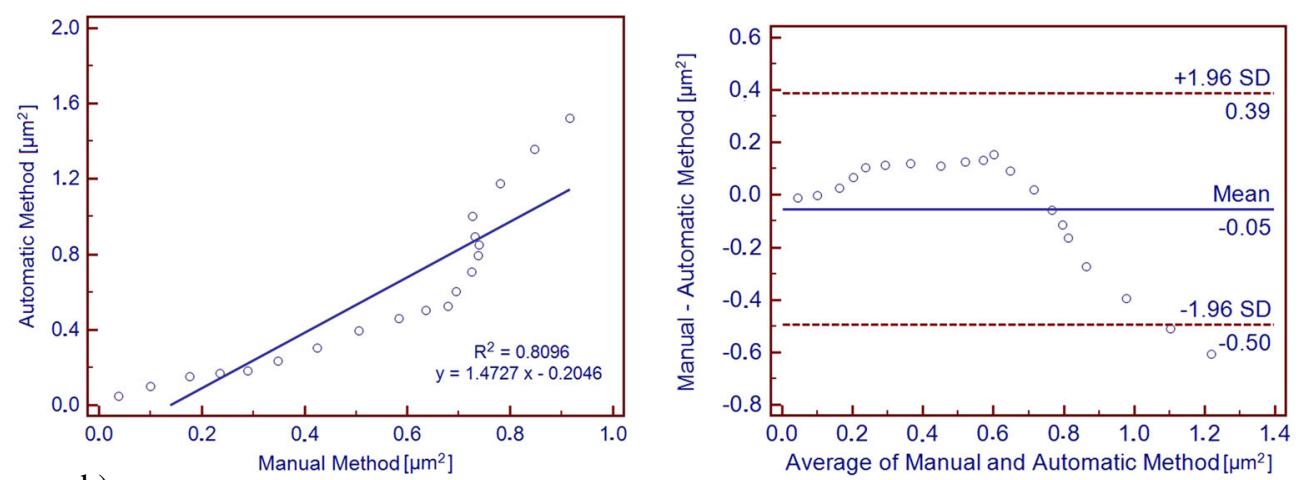

b)

Figure 6. Bland-Altman analysis for comparison of manual and automatic measurements: (a) RBC with the best correlation coefficient and (b) RBC with the worst correlation coefficient.

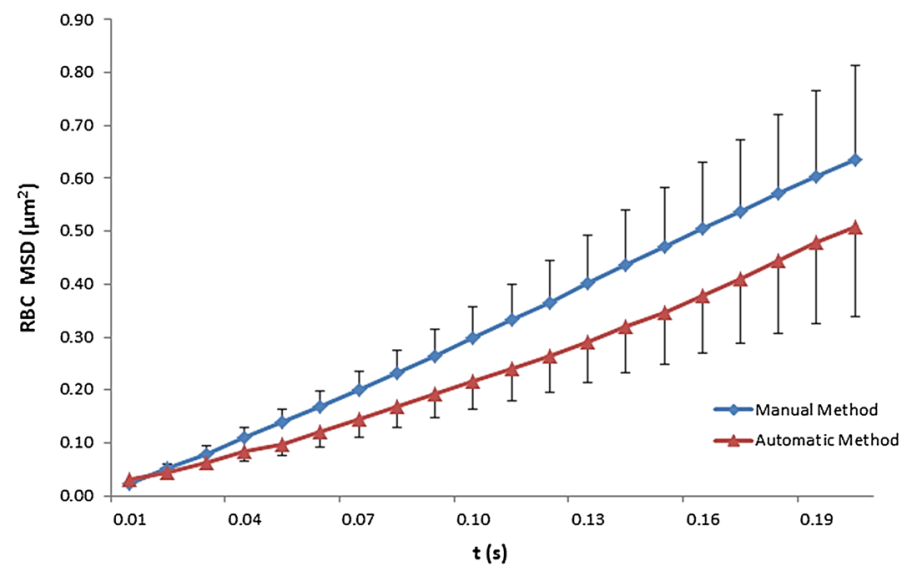

Figure 7. Average of the MSD of 22 RBCs with $95 \%$ confidence level.

Additionally, the ensemble average of the MSD of 22 labeled RBCs was also calculated by using both manual and automatic methods. Figure 7 shows the average MSD of 22 RBCs for both methods whereas Figure 8 shows the corresponding linear regression and Bland-Altman analysis.

The results show not only a good agreement between the two methods (correlation coefficient of 0.9945) but also no significant difference between the two methods. Therefore, these latter results are consistent and reinforce the measurements previously obtained for each individual RBC and as a result it demonstrates that our proposed automatic method is a rapid and reliable technique to calculate several physical quantities such as RBC radial displacement and correspondent MSD. 

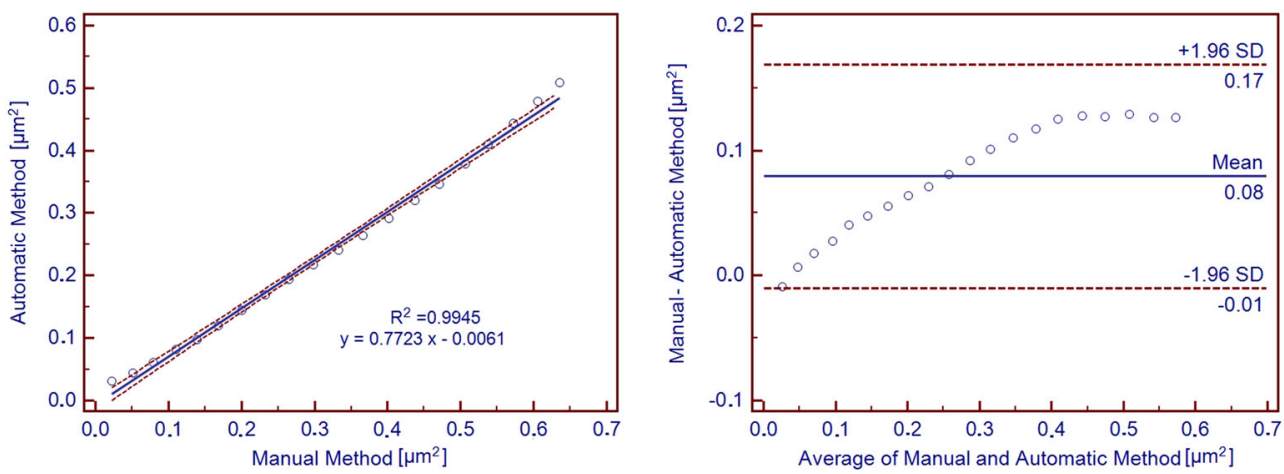

Figure 8. Linear regression and Bland-Altman analysis of MSD of RBC.

During the last decade several automatic particle methods have been proposed to measure trajectories of different kinds of objects. A promising method is the 'Particletracker 2D and 3D' [15], which is a plug-in for Image J. We have analysed our images containing labeled RBCs flowing within the focal plane of interest. After setting the parameters for the RBCs detection, it was possible to obtain automatically the RBC trajectories. However, this method also tracked other artifacts and RBCs flowing in out of focus planes and consequently these results are most of the times extremely chaotic and difficult to be analysed. In contrast our proposed method is able not only to track the RBCs of interest but also to eliminate most of the noise and artifacts that exists in our images. It is worth noting that the proposed automatic method can measure accurately the motion of individual RBCs flowing in Hcts up to $12 \%$. In the near future, further studies with higher Hcts need to be performed to evaluate the possibility of applying the proposed method to measure blood cells flowing in in vivo environments.

\section{CONCLUSIONS}

The present study compared two different methods of tracking RBCs flowing through a $100-\mu \mathrm{m}$ glass capillary, that is, a manual and an automatic method. The proposed automatic method is based on a threshold algorithm and its main purpose is to provide a rapid and accurate way to compute automatically multiple $\mathrm{RBC}$ trajectories. The comparison of the automatic measurements with results obtained by the manual method was made and the results have shown not only a good agreement but also no significant difference between the two methods. Therefore, the proposed automatic method can be successfully integrated with a confocal system and used for fast and accurate RBC measurements. This combination will provide a powerful way to investigate several microcirculation phenomena in a rapid and reliable way. It is worth noting that the accuracy of the proposed automatic method depends strongly on the image quality of the experimental conditions. Therefore, detailed studies with different Hcts need to be performed in the near future because the Hct strongly affects the quality of the images.

\section{ACKNOWLEDGEMENTS}

The authors acknowledge the financial support provided by: Student Mobility Placements with the program Lifelong Learning(Erasmus Program), PTDC/SAU-BEB/108728/2008, PTDC/SAU-BEB/105650/2008, PTDC/EME-MFE/099109/2008 and PTDC/SAU-ENB/116929/2010 from the FCT (Science and Technology Foundation) and COMPETE, Portugal.

\section{REFERENCES}

1. Lima R, Ishikawa T, Imai Y, Takeda M, Wada S, Yamaguchi T. Measurement of individual red blood cell motions under high hematocrit conditions using a confocal micro-PTV system. Annals of Biomedical Engineering 2009; 37:1546-1559. 
2. Crowl LM, Fogelson AL. Computational model of whole blood exhibiting lateral platelet motion induced by red blood cells. International Journal for Numerical Methods in Biomedical Engineering 2009; 26:471-487.

3. Kamada H, Tsubota K, Nakamura M, Wada S, Ishikawa T, Yamaguchi T. A three-dimensional particle simulation of the formation and collapse of a primary thrombus. International Journal for Numerical Methods in Biomedical Engineering 2010; 26:488-500.

4. Yamaguchi T, Ishikawa T, Tsubota K, Imai Y, Nakamura M, Fukui T. Computational blood flow analysis - new trends and methods. Journal of Biomechanical Science and Enginneering 2006; 1:29-50.

5. Lima R, Ishikawa T, Imai Y, Yamaguchi T. Confocal micro-PIV/PTV measurements of the blood flow in micro-channels. In Nano and Micro Flow Systems for Bioanalysis, Collins MW, Koenig CS (eds). Springer: New York, 2012; 131-152.

6. Pinho D, Pereira A, Lima R. Red blood cells motion in a glass microchannel. Numerical Analysis and Applied Mathematics 2010; 1281:963-966.

7. Goldsmith H, Turitto V. Rheological aspects of thrombosis and haemostasis: basic principles and applications. ICTH-Report-Subcommittee on Rheology of the International Committee on Thrombosis and Haemostasis. Journal of Thrombosis and Haemostasis 1986; 55(3):415-435.

8. Lima R. Analysis of the blood flow behavior through microchannels by aconfocal micro-PIV/PTV system. $P h D$ (Eng), Bioengineering and RoboticsDepartment, Tohoku University, Sendai, Japan, 2007.

9. Lima R, Ishikawa T, Imai Y, Yamaguchi T. Blood flow behavior in microchannels: advances and future trends. In Single and two-Phase Flows on Chemical and Biomedical Engineering, Dias R, Martins AA, Lima R, Mata TM (eds). Bentham Science Publishers: Netherlands, 2012; 513-547.

10. Nakano A, Sugii Y, Minamiyama M, Niimi H. Measurement of red cell velocity in microvessels using particle image velocimetry (PIV). Clinical Hemorheology and Microcirculation 2003; 29:445-455.

11. Sugii Y, Okuda R, Okamoto K, Madarame H. Velocity measurement of both red blood cells and plasma of in vitro blood flow using high-speed micro PIV technique. Measurement Science and Technology 2005; 16:1126-1130.

12. Vennemann P, Kiger K, Lindken R, Groenendijk B, Stekelenburg-de Vos S, Hagen T, Ursem N, RPoelmann R, Westerweel J, Hierk B. In vivo micro particle image velocimetry measurements of blood-plasma in the embryonic avian heart. Journal of Biomechanics 2006; 39:1191-1200.

13. Wereley ST, Meinhart CD. Recent advances in micro-particle image velocimetry. Annual Review of Fluid Mechanics 2010; 42:557-576.

14. Adrian R. Particle-imaging techniques for experimental fluid mechanics. Annual Review of Fluid Mechanics 1991; 23:261-304.

15. Sbalzarini IF, Koumoutsakos P. Feature point tracking and trajectory analysis for video imaging in cell biology. Journal of Structural Biology 2005; 151(2):182-195.

16. Machin M, Santomaso A, Mazzucato M, Cozzi MR, Battiston M, Marco L, Canu P. Single particle tracking across sequences of microscopical images: Application to platelet adhesion under flow. Annals of Biomedical Engineering 2006; 34(5):833-846. DOI: 10.1007/s10439-006-9086-8.

17. Acton ST, Wethmar K, Ley K. Brief Communication: Automatic trackingof rolling leukocytes in vivo. Microvascular Research 2002; 63:139-148. DOI: 10.1006/mvre.2001.2373.

18. Lima R, Wada S, Tsubota K, Yamaguchi T. Confocal micro-PIV measurements of three dimensional profiles of cell suspension flow in a square microchannel. Measurement Science and Technology 2006; 17:797-808.

19. Lima R, Wada S, Takeda M, Tsubota K, Yamaguchi T. In vitro confocal micro-PIV measurements of blood flow in a square microchannel: the effect of the haematocrit on instantaneous velocity profiles. Journal of Biomechanics 2007; 40:2752-2757.

20. Lima R, Ishikawa T, Imai Y, Takeda M, Wada S, Yamaguchi T. Radial dispersion of red blood cells in blood flowing through glass capillaries: role of heamatocrit and geometry. Journal of Biomechanics 2008a; 44:2188-2196.

21. Lima R, Wada S, Tanaka S, Takeda M, Ishikawa T, Tsubota K, Imai Y, Yamaguchi T. In vitro blood flow in a rectangular PDMS microchannel: experimental observations using a confocal micro-PIV system. Biomedical Microdevices 2008b; 10(2):153-167.

22. Lima R, Oliveira MSN, Ishikawa T, Kaji H, Tanaka S, Nishizawa M, Yamaguchi T. Axisymmetric PDMS microchannels for in vitro haemodynamics studies. Biofabrication 2009d; 1(3):035005.

23. Fujiwara H, Ishikawa T, Lima R, Matsuki N, Imai Y, Kaji H, Nishizawa M, Yamaguchi T. Red blood cell motions in a high hematocrit blood flowing through a stenosed micro-channel. Journal of Biomechanics 2009; 42:838-843.

24. Yaginuma T, Oliveira MSN, Lima R, Ishikawa T, Yamaguchi T. Red blood cell deformation in flows through a PDMS hyperbolic microchannel. Proceedings of TechConnect World 2011 - Microtech Conference and Expo, Boston, MA, USA, 2011; 2, 505.

25. Yaginuma T, Oliveira MSN, Lima R, Dias R, Ishikawa T, Imai Y, Yamaguchi T. Flow visualization of trace particles and red blood cells in a microchannel with a diverging and converging bifurcation. ECCOMAS Thematic Conference on Computational Vision and Medical Image Processing, Olhão, Portugal, 2012; 209-211.

26. Leble V, Lima R, Dias R, Fernandes C, Ishikawa T, Imai Y, Yamaguchi T. Asymmetry of red blood cell motions in a microchannel with a diverging and converging bifurcation. Biomicrofluidics 2011; 5:044120.

27. Lima R, Fernandes C, Dias R, Ishikawa T, Imai Y, Yamaguchi T. Microscale flow dynamics of red blood cells in microchannels: an experimental and numerical analysis. In Computational Vision and Medical Image Processing: Recent Trends, Vol. 19, Tavares, Jorge (eds). Springer: New York, London, 2011; 297-309. 
28. Abramoff M, Magelhaes P, Ram S. Image processing with image. International Journal of Biophotonics 2004; 11:36-42.

29. MatLab. The MathWorks, Inc., version 7.11. Image Processing Toolbox version 7.1 User's guide, 2010.

30. Rogers SS, Zhao TAZ, Lu JR. Precise particle tracking against a complicated background: polynomial fitting with Gaussian weight. Physical Biology 2007; 4:220-222.

31. Carter BC, Shubeita GT, Gross SP. Tracking single particles: a user-friendly quantitative evaluation. Physical Biology $2005 ; 2: 60-72$.

32. Crocker JC, Grier DG. Methods of digital video microscopy for colloidal studies. Journal of Colloid and Interface Science 1996; 179:298.

33. Eddins SL, Gonzalez RC, Woods RE. Digital Image Processing Using Matlab, 2002.

34. Monteiro FJC. Region - based spatial and temporal image segmentation. PhD Thesis, Faculdade de Engenharia da Universidadedo Porto, 2007.

35. Aly AA, Deris SB, Zaki N. Research review for digital image Segmentation techniques. International Journal of Computer Science \& Information Technology 2011; 3(5):99-106. DOI: 10.5121/ijcsit.2011.3509 99.

36. Pham DL, Xu C, Prince JL. Current methods in medical image segmentation. Annual Review of Biomedical Engineering 2000; 2:315-337.

37. Bovik A. The essential Guide to Image Processing. Elsevier: Burlington, MA, 2009.

38. Pinho D. Determination and characterization of red blood cells trajectories: a semi-automatic method. Master in Biomedical Technology, Polytechnic Institute of Bragança, Portugal, 2011. (in portuguese).

39. Meijering E, Smal I, Danuser G. Tracking in molecular bioimaging. IEEE Signal Processing Magazine 2006; 23:46-53.

40. Kim S, Kong RL, Popel AS, Intaglietta M, Jonhson PC. A computer - based for determination of cell-free layer width in microcirculation. Microcirculation 2006; 13:199-207. DOI: 10.1080/10739680600556878.

41. Namgung B, Ong PK, Wong YH, Lim D, Chun KC, Kim S. A comparative study of histogram-based thresholding of cell-free layer wisth in small blood vessels. Physiological Measurement 2010; 31:N61-N70.

42. MedCalc Software, Version 12.1.4 Broekstraat 52, 9030 Mariakerke, Belgium.

43. Bland JM, Altman DG. Statistical methods for assessing agreement between two methods of clinical measurement. Lancet 1986; i:307-310. 